Planetary Systems in the Universe - Observation, Formation and Evolution

Proceedings IAU Symposium No. 202, (C)2004 IAU

Alan Penny, Pawel Artymowicz, Anne-Marie Lagrange, $\mathcal{G}$ Sara Russell, eds.

\title{
Stable mass ranges of $v$ Andromedae planetary system
}

\author{
Takashi Ito \\ Astronomical Data Analysis Computer Center, National Astronomical \\ Observatory, Mitaka, Tokyo 181-8588, Japan
}

\author{
Shoken M. Miyama \\ Theoretical Astrophysics Division, National Astronomical Observatory, \\ Mitaka, Tokyo 181-8588, Japan
}

\begin{abstract}
Doppler observation of extrasolar planets through the radial velocity displacement of their host stars can only determine lower limits of planetary masses. We numerically integrate $v$ Andromedae planetary orbits with various initial conditions of masses and angle variables to investigate which initial configuration produces stable orbits during the timescale of host star's age. According to our preliminary results starting from Lick dataset, $v$ Andromedae planetary system seems to remain stable over the timescale of its host star's age if $\sin i \geq 0.7$ where $i$ is the unknown line-of-sight inclination of planetary orbits. In this case we may estimate that the upper limit masses of $v$ Andromedae planets in our model is about $1 / 0.7 \sim 1.43$ times larger than its minimum.
\end{abstract}

\section{Introduction, numerical models, and methods}

Among the several methods to detect extrasolar planets, Doppler observation of the radial velocity displacement of central stars is currently most powerful and popular technique. Most of extrasolar planets have been detected by the Doppler observation. Three planets around $v$ Andromedae have been discovered also by the Doppler observation technique (Butler et al. 1999).

However, since the Doppler measurement can only determine a product $m \sin i$ (where $m$ is the planetary mass and $i$ is the inclination between the lineof-sight of observers and a normal to the orbital plane), planetary mass $m$ itself cannot be determined uniquely except for its lower limit, i.e. when $\sin i=1$.

This inherent uncertainty included in the Doppler observation may be somewhat supplemented by numerical integrations of planetary orbital motions because: (1) planetary systems which contain more than one planet generally becomes unstable within finite timescales, and (2) this instability timescale becomes shorter when gravitational interaction among planets is stronger: for example, when the planetary masses are larger. Thus we may possibly estimate stable mass range of $v$ Andromedae planets by a number of numerical integrations in which we vary the planetary masses from small ones to large ones. Out of the stable mass range, instability timescale of the system becomes shorter than the timescale of $v$ Andromedae host star's age, $\sim 2.6 \times 10^{9}$ years.

In our calculation, we only take outer two planets (c and d) on coplanar orbits into account to save the amounts of numerical computation. We supple- 
ment our omission of the innermost planet (b) by incorporation of a circular ring potential which represents the time-averaged effect of the omitted planet. All the orbital elements and masses used in our integrations are adopted from Butler et al. (1999). Fixing initial eccentricities $e$ and orbital periods $P$ as their nominal values, we vary longitudes of pericenter $(\varpi)$ and mean anomalies randomly within their observational uncertainties.

We define the instability time $T_{I}$ as being the time elapsing between the initial configuration and the final unstable configuration (ejection or collision of planets) in numerical calculations. Final numerical results are gathered and plotted on $\left(\sin i, T_{I}\right)$ diagram. 20 to 30 integrations per one $\sin i$ bin are performed using different initial orbital elements from $\sin i=0.4$ to $\sin i=1.0$ stepping 0.1. All the integrations progress to future direction in time.

\section{Stable mass range of $v$ Andromedae planets and orbital alignment}
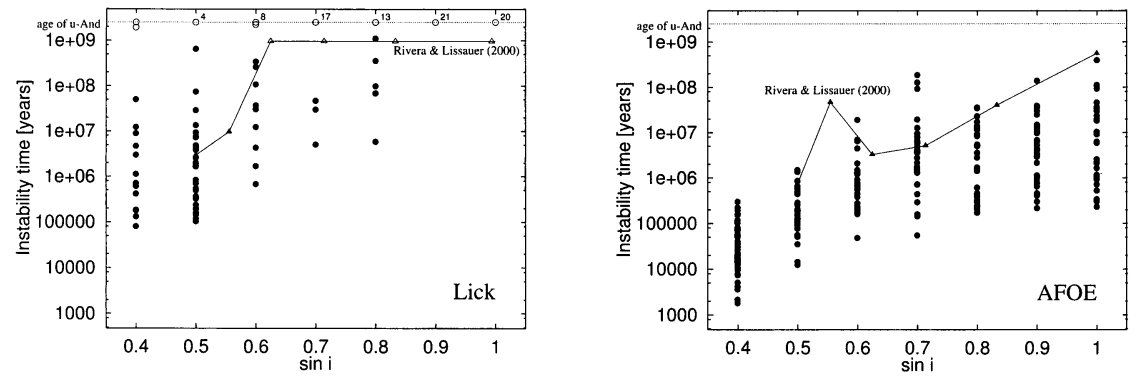

Figure 1. ( $\left.\sin i, T_{I}\right)$ diagram for numerical integrations using Lick (left) and AFOE (right) datasets. Our results are represented by filled and open circles. Numbers located rightside at the open circles denote that the results of several integrations are overlapped at the open circles.

Results of our numerical integrations for two datasets (Lick and AFOE) are summarized in Figure 1. Note that all the open symbols indicate that although the integrations have been stopped there for certain reasons, the systems were still stable; instability times $T_{I}$ should be much longer than actually shown by the open symbols there. Results from Rivera and Lissauer (2000) are also plotted for comparison (filled and open triangles).

We can find four major viewpoints in Figure 1. (i) Instability times $T_{I}$ are lengthen exponentially as $\sin i$ increases. (ii) There is a wide variety in instability times $T_{I}$, several orders of magnitude, especially when $\sin i$ is small. It reflects the chaotic character of this planetary system as pointed out by Laughlin and Adams (1999). (iii) When using Lick dataset, most integrations in $\sin i \leq 0.6$ region are likely to become unstable before they reach the timescale of the host star's age. When $\sin i \geq 0.7$, there are much more open circles than filled ones which indicates that the system are likely to remain stable in most cases. Although the number of integration is still not enough to derive definite statistical conclusions, the upper limit of $v$ Andromedae planetary masses in our model may be set as $1 / 0.7 \simeq 1.43$ times larger than their minimum. (iv) When using 
AFOE dataset, all integrations became unstable before they reached the host star's age. It is probably due to the larger nominal eccentricity and mass of the outermost planet (d) in AFOE dataset compared with Lick dataset.
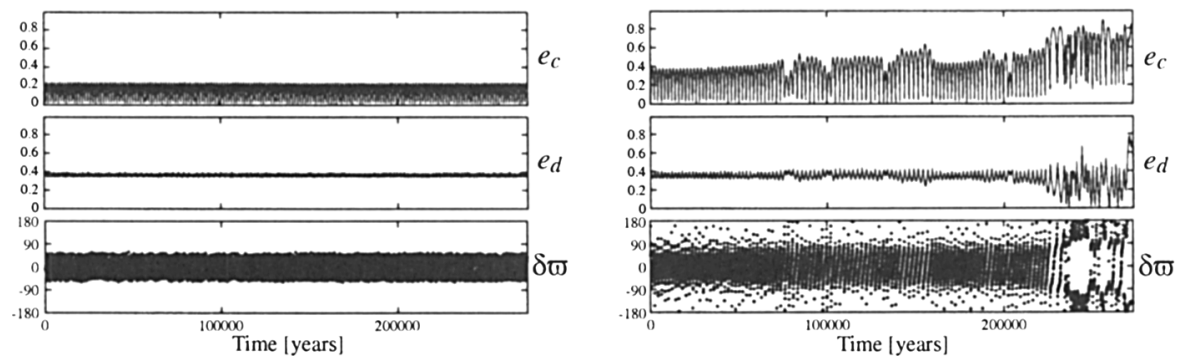

Figure 2. (Left) An example of eccentricities $\left(e_{c}, e_{d}\right)$ and difference in $\varpi$ $\left(\delta \varpi=\varpi_{c}-\varpi_{d}\right)$ of the two planets using Lick dataset with $\sin i=0.4$. Initial orbital elements are $e_{c}=0.23, \varpi_{c}=222.7^{\circ}, e_{d}=0.36, \varpi_{d}=234.7^{\circ}$. (Right) Another example which started from different initial orbital elements as $e_{c}=0.23, \varpi_{c}=275.2^{\circ}, e_{d}=0.36, \varpi_{d}=232.3^{\circ}$.

Large diversity of instability times $T_{I}$ in Figure 1 can be better understood by viewing the evolution of planetary orbital elements. In Figure 2, eccentricities and longitudes of pericenter of the planets in two integrations in Figure 1 are shown. One integration (right panels) was terminated after about $2.7 \times 10^{5}$ years because the inner planet (c) has been ejected out of the system, while the other (left panels) remained stable for several $10^{8}$ years.

A major reason of such large difference in orbital evolutions in above two cases is alignment of longitudes of pericenter. In many of stable orbits in Figure 1 using Lick dataset, pericenters of the two planets align: $\delta \varpi=\varpi_{c}-\varpi_{d}$ is kept small, which can help the planets to avoid close encounters. Small difference in $\delta \varpi$ in the above two integrations finally leads to the large difference in their orbital evolution and stability. An analytical explanation on this orbital alignment is described in Kinoshita and Nakai (2000).

Acknowledgments. This study was supported by the ADAC scientific simulation project, National Astronomical Observatory of Japan (dti02-1999).

\section{References}

Butler, R.P., Marcy, G.W., Fischer, D.A., Brown, T.W., Contos, A.R., Korzennik, S.G., Nisenson, P., \& Noyes, R.W. 1999, ApJ, 526, 916

Kinoshita, H. \& Nakai, H., 2000 , PASP Conf. Ser., this volume

Laughlin, G. \& Adams, F.C. 1999, ApJ, 526, 881

Rivera, E.J. \& Lissauer, J.J. 2000, ApJ, 530, 454 